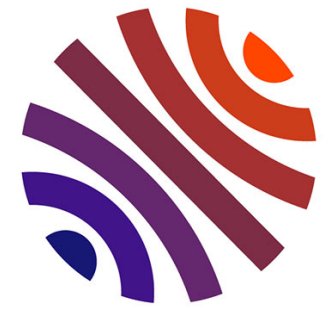 \\ HAL open science
}

\section{Lombalgie et prise en charge hiérarchisée : une obligation démographique}

Audrey Petit, Elsa Parot-Schinkel, Natacha Fouquet, Isabelle Richard, Yves Roquelaure

\section{- To cite this version:}

Audrey Petit, Elsa Parot-Schinkel, Natacha Fouquet, Isabelle Richard, Yves Roquelaure. Lombalgie et prise en charge hiérarchisée : une obligation démographique. Revue du Rhumatisme, 2011, 78 (1), pp.95 - 96. 10.1016/j.rhum.2010.08.012 . hal-03389893

\section{HAL Id: hal-03389893 \\ https://univ-angers.hal.science/hal-03389893}

Submitted on 21 Oct 2021

HAL is a multi-disciplinary open access archive for the deposit and dissemination of scientific research documents, whether they are published or not. The documents may come from teaching and research institutions in France or abroad, or from public or private research centers.
L'archive ouverte pluridisciplinaire HAL, est destinée au dépôt et à la diffusion de documents scientifiques de niveau recherche, publiés ou non, émanant des établissements d'enseignement et de recherche français ou étrangers, des laboratoires publics ou privés. 


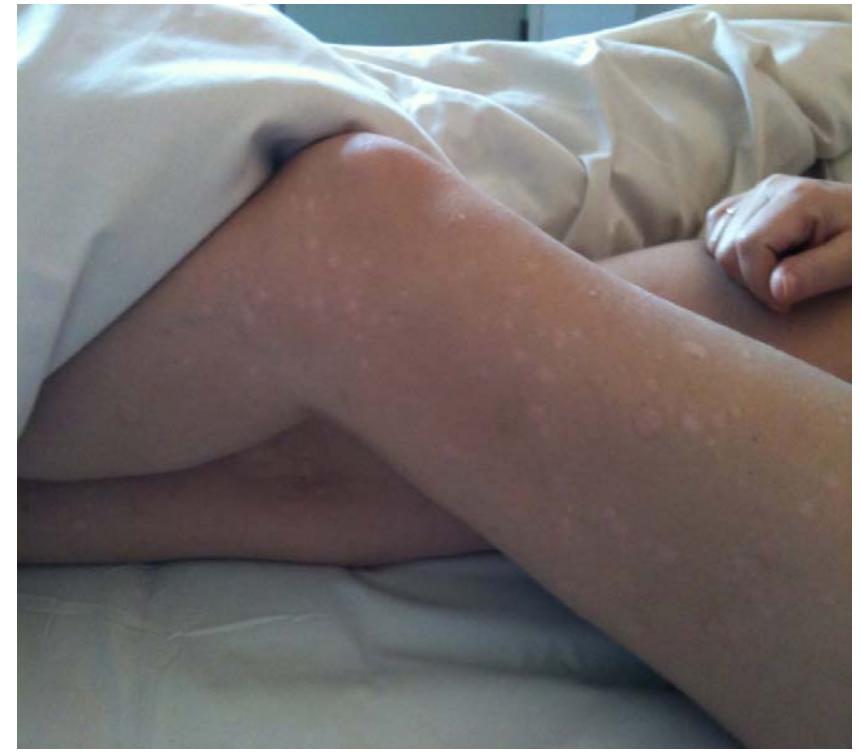

Fig. 1. Plaques nummulaires à base érythémateuse, bien limitées, discrètement squameuses.

l'abatacept est retenue devant un antécédent de psoriasis blanchi et l'évolution favorable des lésions cutanées après son arrêt. Le mécanisme de réactions cutanées paradoxales sous anti-TNF est discuté. Plusieurs mécanismes semblent intriqués : hypothèse infectieuse, hyperproduction d'interferon $\alpha$ induite sous anti-TNF déclenchant des réactions psoriasiformes, l'activation des Th17 et une inhibition des Treg [5,10]. Dans notre cas, le rôle potentiel d'un déséquilibre cytokinique et d'une augmentation paradoxale des médiateurs de l'inflammation induits par les anti-TNF et l'abatacept peut être une hypothèse évoquée dans la réactivation du psoriasis.

\section{Conflit d'intérêt}

Les auteurs ne déclarent aucun conflit d'intérêt.

\section{Références}

[1] Cañete JD, Celis R, Hernández V, et al. Synovial immunopathological changes associated with successful abatacept therapy in a case of severe refractory psoriatic arthritis. Ann Rheum Dis 2010;69:935-6.

[2] Vieira FJ, Callado MR, Vieira WP. Abatacept as an option therapy in difficult to treat psoriatic arthritis. Rheumatol Int 2010;30:849-50.

[3] Abrams JR, Lebwohl MG, Guzzo CA, et al. CTLA4Ig-mediated blockade of T-cell co-stimulation in patients with psoriasis vulgaris. J Clin Invest 1999; 103:1243-52.

[4] Olivieri I, D’Angelo S, Mennillo GA et al. Abatacept in spondyloarthritis refractory to tumour necrosis factor alpha inhibition. Ann Rheum Dis 2009;68:151-2.

[5] Wendling D, Balblanc JC, Briançon D, et al. Onset or exacerbation of cutaneous psoriasis during TNF-alpha antagonist therapy. Joint Bone Spine 2008;75:315-8.

[6] Roux $\mathrm{CH}$, Brocq O, Leccia N, et al. New onset psoriatic palmoplantaris pustulosis following infliximab therapy: a class effect? J Rheumatol 2007;34:434-7.

[7] Kary S, Worm M, Audring H, et al. New onset or exacerbation of psoriatic skin lesions in patients with definite rheumatoid arthritis receiving tumour necrosis factor alpha antagonists. Ann Rheum Dis 2006;65:405-7.

[8] Chaby G, Andréjak M, Lok C, et al. Psoriasis en goutte induit par étanercept récidivant sous abatacept. Ann Dermatol Venereol 2009;136(12 Suppl. 1):A321.

[9] Sibilia J, Westhovens R. Safety of T-cell co-stimulation modulation with abatacept in patients with rheumatoid arthritis. Clin Exp Rheumatol 2007;25(5 Suppl. 46):S46-56.

[10] Ma HL, Napierata L, Stedman N, et al. Tumor necrosis factor alpha blockade exacerbates murine psoriasis-like disease by enhancing Th17 function and decreasing expansion of Treg cells. Arthritis Rheum 2010;62: 430-40.

Amélie Florent ${ }^{\mathrm{a}, *}$ Christine Albert ${ }^{\mathrm{a}}$ Damien Giacchero ${ }^{\mathrm{b}}$
Christian Roux ${ }^{\mathrm{a}}$

Liana Euller-Ziegler ${ }^{\text {a }}$

a Service de rhumatologie Pr-Euller-Ziegler, hôpital de l'Archet-1, CHU de Nice, 151, route Saint-Antoine de Ginestière, BP 3079, 06202 Nice cedex 3, France

b Service de dermatologie-vénéréologie, hôpital de l'Archet-2, CHU de Nice, 151, route Saint-Antoine de Ginestière, BP 3079, 06202 Nice cedex 3, France

* Auteur correspondant. Adresse e-mail : amelie.florent@orange.fr (A. Florent).

5 août 2010

Disponible sur Internet le 24 septembre 2010 doi:10.1016/j.rhum.2010.08.007

\section{Lombalgie et prise en charge hiérarchisée: une obligation démographique}

\section{Mots clés :}

Lombalgie chronique

Épidémiologie

Travail

Trouble musculo-squelettique

Prise en charge

Dans les pays industrialisés, la lombalgie chronique constitue l'affection bénigne la plus coûteuse dans la population active. En effet, l'absentéisme et l'invalidité liés à la lombalgie présentent un pic entre 30 et 50 ans touchant une population jeune en phase d'ascension professionnelle [1,2].

Selon les dernières données de la surveillance épidémiologique des troubles musculo-squelettiques (TMS) dans la région des Pays de la Loire, le nombre de salariés souffrant du bas du dos est colossal : plus de 268,000 salariés ont eu mal au cours des sept derniers jours et plus d'un demi million au cours des 12 mois précédant l'enquête (Tableau 1).

Sachant qu'un peu moins de $10 \%$ des lombalgies mécaniques sont à risque de devenir chroniques, cela concernerait 50,000 salariés. Et si l'on considère que $10 \%$ de ces patients seront lourdement handicapés et à risque de désinsertion socioprofessionnelle, cela revient à dire qu'environ 5000 patients nécessiteront une prise en charge de type restauration fonctionnelle du rachis (RFR) par an dans les Pays de la Loire [3].

Les programmes de RFR mis en place en France depuis les années 1990 ne sont donc pas en adéquation avec de si grandes populations de patients. De plus, même s'ils ont fait la preuve de leur efficacité sur l'amélioration fonctionnelle et la reprise du travail à court et moyen terme, il n'en demeure pas moins que ces programmes représentent un coût significatif pour la communauté [4].

D’où la nécessité d'une prise ne charge hiérarchisée pour :

- d'une part, développer des stratégies adaptées au profil d'évolution des patients en formant les patriciens de soins pri-

Tableau 1

Lombalgie chez les salariés des Pays de la Loire ${ }^{a}$.

\begin{tabular}{lll}
\hline & $\begin{array}{l}\text { Symptômes au cours } \\
\text { des 12 derniers mois }\end{array}$ & $\begin{array}{l}\text { Symptômes au cours } \\
\text { des 7 derniers jours }\end{array}$ \\
\hline Femmes & 244,253 & 118,503 \\
Hommes & 316,552 & 150,059 \\
Total & 560,805 & 268,561 \\
\hline
\end{tabular}

a Sources : institut de Veille Sanitaire (InVS), Saint-Maurice, France et laboratoire d'épidémiologie et d'ergonomie en santé au travail (LEEST) - unité associée InVS, université d'Angers, France. 
maires pour qu'ils adaptent leur pratique. C'est tout l'intérêt d'une prise en charge précoce, soit avant le stade de chronicité, et l'influence de la préférence des patients quant au succès des traitements proposés. Il est admis qu'une intervention précoce au stade de lombalgie persistante (au-delà de six semaines) laisse envisager la possibilité d'une prévention du passage à la chronicité [5-7];

- d'autre part, identifier les patients ayant un mauvais pronostic et relevant de traitements ciblés et de complexité croissante avec une prise en charge bio-psycho-sociale adaptée. Les dernières recommandations internationales [5] confortent l'intérêt des programmes de rééducation à composante physique et psychologique pouvant intégrer des mesures d'ordre social et professionnel réalisée sur plusieurs semaines représentant 100 heures de traitement ou plus. Mais elles doivent être réservées aux patients en situation de handicap lourd et d'échec.

Notre équipe a démontré l'efficacité d'une prise en charge des patients lombalgiques chroniques en kinésithérapie libérale. De même, plusieurs établissements proposent des programmes dits "légers " (mini-RFR) dont l'efficacité, à un coût bien moindre, par rapport aux stages «standards » n'est pas négligeable [8,9]. L'enjeu est donc de distinguer une population par rapport à une autre et d'adapter au mieux les traitements.

Si l'on intègre que la prévalence des lombalgies chroniques a été multipliée par 2,6 au cours des 15 dernières années et que le degré d'incapacité générée par individu atteint reste stable avec un recours aux soins en augmentation [10], il est urgent de repenser nos stratégies de prise en charge.

\section{Conflit d'intérêt}

Les auteurs ne déclarent aucun conflit d'intérêt.

\section{Références}

[1] Rossignol M, Rozenberg S, Leclerc A. Epidemiology of low back pain: what's new? Joint Bone Spine 2009;76:608-13.

[2] Frymoyer JW, Cats baril WL. An overview of the incidences and cost of low back pain. Orthop Clin North Am 1991;22:263-71.

[3] Anderson GB. Epidemiological features of chronic low back pain. Lancet 1999;354:581-5.

[4] Poireaudeau S, Rannou F, Lefevre-Colau MM, et al. Rehabilitation on effort of low back pain. Functional restoration programs. Presse Med 2004;33:413-8.

[5] Chou R, Loeser JD, Owens DK, et al. Interventional therapies, surgery, and interdisciplinary rehabilitation for low back pain. An evidence-based clinical practice guideline from the American pain society. Spine 2009;34:1066-77.

[6] Savigny P, Watson P, Underwood M, et al. Early management of persistent nonspecific low back pain: summary of NICE guidance. BMJ 2009;338:1441-2.

[7] Mayor S. NICE recommends early intensive management of persistent low back pain. BMJ 2009;338:1289.

[8] Jousset N, Fanello S, Bontoux L, et al. Effects of functional restoration versus $3 \mathrm{~h}$ per week physical therapy: a randomized controlled study. Spine 2004;29:487-93.

[9] Roche G, Ponthieux A, Parot-Shinkel E, et al. Comparison of a Functional Restoration Program with Active Individual Physical Therapy for patients with chronic low back pain. Arch Phys Med Rehabil 2007;88:1229-35.

[10] Freburger JK, Holmes GM, Agans RP, et al. The rising prevalence of chronic low back pain. Arch Int Med 2009;169:251-8.

Audrey Petit Le Manac'h ${ }^{a, b, *}$ Elsa Parot-Schinkel ${ }^{b, c}$ Natacha Fouquet ${ }^{b, d}$ Isabelle Richard ${ }^{\mathrm{b}, \mathrm{e}}$ Yves Roquelaure ${ }^{\mathrm{a}, \mathrm{b}}$

a Service de médecine interne et pathologie professionnelles, CHU d'Angers, Angers, France

b Laboratoire d'épidémiologie et ergonomie en santé au travail (LEEST), unité associée InVS, université d'Angers, 4, rue Larrey, 49933 Angers cedex, France c Département de santé publique, CHU d'Angers, Angers, France

${ }^{\mathrm{d}}$ Institut de Veille Sanitaire (InVS), Saint-Maurice,

France

e Service de médecine physique et réadaptation, $\mathrm{CHU}$

d'Angers, Angers, France

* Auteur correspondant. Adresse e-mail : aupetit@chu-angers.fr

(A. Petit Le Manac'h).

19 août 2010

Disponible sur Internet le 8 octobre 2010

doi:10.1016/j.rhum.2010.08.012

Syndrome FATCO-aplasie fibulaire, campomélie tibiale et oligosyndactylie

Mots clés :

Aplasie fibulaire

Campomélie tibiale et oligosyndactylie

Syndrome FATCO

Syndrome aplasie-ectrodactylie

Les auteurs rapportent un cas d'aplasie fibulaire, de campomélie tibiale et d'oligodactylie (FATCO) chez un nouveau-né de sexe masculin. La grossesse et l'accouchement se sont déroulés normalement, à l'exception de la survenue de deux chutes rapportées par la mère deux mois avant l'accouchement sans signe, ni symptômes de traumatisme ou hospitalisation. La mère était âgée de 36 ans et le père de 45 ans, en bonne santé, de même que leurs deux enfants (un garçon et une fille). Il n'existait pas d'antécédent familial de malformation congénitale squelettique.

La malformation congénitale du membre inférieur gauche a été identifiée après la naissance : raccourcissement, angulation antérieure du milieu du tibia avec une dépression cutanée en regard. Seulement, quatre orteils déviés latéralement étaient présents au membre inférieur gauche (absence du troisième rayon distal). Il n'existait pas d'autres anomalies somatiques ou neurologiques ou de dysmorphie faciale.

Les radiographies du membre inférieur gauche ont montré un raccourcissement marqué du tibia et une angulation dans la direction ventrale. Le noyau d'ossification de l'extrémité proximale du tibia était absent. Il existait une aplasie fibulaire, une hypoplasie du calcanéum et une oligodactylie (absence du troisième rayon) (Fig. 1 et 2). Les marqueurs du métabolisme osseux étaient normaux. Après sa sortie d'hospitalisation, le patient a suivi une rééducation et une chirurgie partielle a été réalisée afin de corriger l'inégalité de longueur des membres inférieurs.

Les malformations des membres surviennent avec une incidence d'un par millier de naissances. La fibula est fréquemment touchée par une agénésie, qui peut être uni- ou bilatérale, rarement familiale, de manière isolée ou dans le cadre de divers syndromes. L'agénésie est le plus souvent unilatérale, isolée et survient rarement sans cause spécifique.

Un syndrome similaire touchant les membres a été décrit par Courtens et al. chez un nouveau-né de sexe masculin [1]. Il présentait un raccourcissement et une angulation du tiers distal du tibia droit avec une dépression de la peau en regard, une aplasie fibulaire, une oligosyndactylie de la jambe droite et une oligosyndactylie de la main gauche. Huber et al. ont décrit des anomalies semblables [2] - angulation et raccourcissement du tibia associés

\footnotetext{
is Ne pas utiliser, pour citation, la référence française de cet article mais sa référence anglaise dans le même volume de Joint Bone Spine (doi:10.1016/j.jbspin.2010.08.013).
} 